\title{
Overview of types of ontology in the software development process
}

\begin{abstract}
Ontology is a word that has been the subject of many studies. It is an important concept in computer science to formally represent knowledge in a way that software can process the knowledge and reason about it. The software engineering ontology assists in defining information for the exchange of semantic project information frameworks. Research into ontological issues has been widely active in various areas. This paper presents the origin of ontology research and gives the different definitions of ontology. The paper provides an overview of ontology and its types including the building and design for an enterprise system. Finally the paper provides a systematic review of the subject.
\end{abstract}

Keyword: Ontology; Types of ontology; Building; Design of ontology; Enterprise system 